\title{
Farmacoepidemiología en el programa de formación de la especialidad de oftalmología en Cuba.
}

\section{Pharmacoepidemiology in the training program for the specialty of ophthalmology in Cuba.}

\author{
Kenia Galindo Reymon", Ana Julia Gracia Milián², Pablo León Cabrera ${ }^{3}$, Iraisis Hormigo Puerta ${ }^{4}$, \\ Susana Marques Villalon ${ }^{5}$
}

1 Médico. MsC Farmacoepidemiología. Especialista de II grado en Oftalmologia y vitreoretina. Profesor asistente e investigador auxiliar. Instituto Nacional de Oftalmología Angola.

2 Médico. Especialista II grado en Farmacología y Administración y Organización de Salud. Doctor en Ciencias de la Salud. Máster en Economía de la Salud. Profesora e Investigadora Titular. Corporación de Salud del Maresma y la Selva. España.

3 Médico. Especialista II grado en Epidemiología y Administración y Organización de Salud. Master en Salud Pública, Dr. en Ciencias. Profesor titular e investigador auxiliar. Dirección Nacional de Salud Pública Angola.

4 Médico. Master en Atención Integral al Adulto Mayor. Especialista de II grado en oftalmología. Profesor e investigador auxiliar. ICO Ramón Pando Ferrer. Cuba.

5 Médico. Master en Longevidad Satisfactoria. Especialista de II grado en oftalmología y Córnea. Profesor auxiliar. Hospital Militar Nacional de Angola.

*Correspondence: Kenia Galindo Reymond e mail: grkenia@gmail.com

Recibido: 24-11-2020; Aceptado: 17-2-2021; Publicado: 17-2-2021

Resumen: Introducción: En la formación del especialista de oftalmología es necesario abordar los temas de farmacoepidemiología para que los residentes en formación puedan adquirir conocimientos y desarrollar competencias que puedan contribuir a la seguridad del paciente durante el proceso de atención. Objetivo: Identificar en el programa de formación del oftalmólogo cubano los contenidos temáticos de farmacoepidemiología y proponer los necesarios en su formación. Metodología: Se realizó un análisis de contenido cualitativo directo del programa de la especialidad utilizando las tres etapas de la metodología de García Milian. Se seleccionaron nueve expertos por criterios de inclusión relevantes (Doctor en Ciencias, con maestría de Farmacoepidemiología y/o con especialidad de oftalmología y categoría de profesor auxiliar) y se definieron los contenidos temáticos a través de la técnica Delphi. Resultados: En el programa de formación del oftalmólogo cubano no se incluyen de forma explícita los contenidos temáticos relacionados con la farmacoepidemiología. Los expertos llegaron al consenso de la necesidad de incorporar siete contenidos temáticos en el programa. Conclusiones: Se evidenció la poca inclusión y sistematización de contenidos temáticos de farmacoepidemiología en la formación del oftalmólogo cubano, los expertos definieron e incluyeron en el currículo de formación de este especialista los contenidos temáticos que puedan contribuir a un mejor desempeño profesional y seguridad del paciente.

Palabras clave: programa de formación, farmacoepidemiología, oftalmología

Abstract: Introduction: In the formation of ophthalmology specialist is necessary to address the
issues of Pharmacoepidemiology for residents in training to acquire knowledge and develop skills
that can contribute to the safety of the patient during care. Objective: To identify the training
program of the Cuban ophthalmologist thematic content of pharmacoepidemiology and propose
necessary in their training. Methodology: A content analysis was performed qualitative direct
specialty program using the three stages of the García Milian as methodology. Nine experts were
selected for inclusion criteria relevant (Doctor of Science, with mastery of Pharmacoepidemiology
and / or specialty and category ophthalmology assistant professor) and content areas defined by
the Delphi technique. Results: During the training program the Cuban ophthalmologist not 
explicitly included the topics related to the pharmaco. The experts reached consensus on the need to incorporate seven thematic content in the program. Conclusions: We showed little inclusion and systematization of thematic content of pharmacoepidemiology in the formation of the Cuban ophthalmologist, experts defined and included in the training curriculum of this specialist content areas that can contribute to improved performance professional and safety of the patient.

Key Words: Training program, pharmacoepidemiology, ophthalmology

\section{Introducción}

La formación de recursos humanos constituye una prioridad para los sistemas de salud en los momentos actuales. Durante este proceso es vital la integración del macro y micro currículo desde su diseño para lograr un profesional capaz de actuar con calidad en diferentes escenarios. En el caso del oftalmólogo, la articulación de los saberes propios de la especialidad con la farmacoepidemiología favorece una formación integral y de excelencia. La farmacoepidemiología permite aplicar los conocimientos, métodos y razonamientos epidemiológicos al estudio de los efectos (beneficiosos o perjudiciales) y los usos de los medicamentos en las poblaciones. Es entendida como una tecnología que estudia la utilización de las opciones terapéuticas y su impacto en el orden médico, económico y social, además posibilita la información útil y oportuna para la toma de decisiones clínica y de gestión en el Sistema de Salud (1).

La formación de los profesionales prescriptores en condiciones de incorporar a su práctica profesional criterios de selección de información para la práctica clínica habitual y el manejo científico de los problemas de salud de sus pacientes, orientados al uso racional de los medicamentos constituye un aspecto básico en los currículos formativos. En las modificaciones que se realizaron al programa de oftalmología en su última revisión no se registran evidencias que traten en el currículo las habilidades y contenidos relacionados con la farmacoepidemiología. Esto tiene una traducción en la escasa producción de investigaciones en esta área de cocimiento relacionadas con la farmacovigilancia y el uso racional de medicamentos. En 2012, el Ministerio de Salud Pública (MINSAP) constituyó la "Comisión Nacional de Competencia y Desempeño" para evaluar el desempeño profesional sobre la base de las competencias laborales en el Sistema Nacional de Salud (SNS). Se inició de forma experimental un proceso de identificación y normalización de las competencias laborales en especialidades médicas, mediante expertos seleccionados por los grupos nacionales de especialidades (2). No existen referencias de que la especialidad de oftalmología estuviera incluida entre las especialidades médicas elegidas. Existen evidencias científicas escasas en el contexto cubano sobre el uso racional de medicamentos y la vigilancia de los fármacos en oftalmología. En la revista cubana de oftalmología desde el 2011 hasta el 2018 en los 29 volúmenes se publicaron 483 trabajos, solo dos de ellos abordan temas relacionados con la farmacoepidemiología (3-4). A esto se le suma que en el tema de la farmacoepidemiología no se aborda en los proyectos de investigación, ni en tesis de especialidad y doctorado. A partir de lo antes planteado surge la necesidad de identificar en el programa de formación del oftalmólogo cubano los contenidos temáticos de farmacoepidemiología, así como formular los contenidos temáticos de esta área del conocimiento al programa.

\section{Métodos}

Se realizó un análisis de contenido cualitativo de tipo directo. Se indagó sobre la presencia del tema de la farmacoepidemiología en el programa, teniendo en cuenta la metodología propuesta por García Milian y colaboradores (5) que permite el análisis y crítica a un programa de estudio. Para la recogida de la información se utilizó la técnica de la revisión documental mediante el análisis del programa de oftalmología. En este proceso se 
realizó la indización o descripción de las características (con palabras clave y con descriptores). En este caso se utilizaron los términos medicamento, farmacoepidemiología, prescripción, seguridad, farmacovigilancia, reacciones adversas y pauta terapéutica. Con posterioridad se procedió con el resumen o descripción sustancial. En este estudio se hizo un resumen mixto y por último se clasificaron los temas incluidos en el programa.

Se trabajó con un grupo de expertos para las definiciones de los contenidos temáticos de farmacoepidemiología mediante la metodología Delphi. La selección de los expertos fue por muestreo intencional y se tuvieron en cuenta criterios como competencia, creatividad, disposición a participar, conformidad, capacidad de análisis, espíritu colectivista y autocrítico. Fueron seleccionados 7 expertos (4 especialistas en Oftalmología y 3 Farmacólogos) con más de 10 años de experiencia laboral, categoría docente principal y categoría científica de Máster y/o Grado de Doctor en Ciencias. Durante las etapas de la técnica Delphi se tuvieron en cuenta los procedimientos de las etapas de preparación, consulta y consenso. En la fase preparatoria se confeccionó un resumen del análisis del programa y se elaboró una guía donde se explicó el procedimiento a utilizar durante la técnica y se presentaron la propuesta de temas y contenidos de farmacoepidemiología a incluir en el programa. Se orientó el análisis de las propuestas de temas y contenidos a partir del programa, se solicitó la evaluación de los contenidos propuestos para lo cual dieron un valor del 0 al 10 a cada uno y se solicitó la posibilidad de inclusión de otras propuestas. Estas informaciones fueron enviadas por correo electrónico. En la segunda etapa se realizó el procesamiento de la información enviada por los expertos, se calculó la media y la desviación estándar de cada uno de los contenidos propuestos por expertos y se clasificaron en una escala donde la categoría de no importante se consideró cuando la media de la puntuación fue entre 0 y 3; importante cuando fue entre 4 y 7 y muy importante cuando fue entre 8 y 10. Los resultados de cada ronda fueron enviados por correo electrónico. Fue considerado el consenso cuando se logró el 80\% como criterio de mayoría. En la etapa de consenso se distribuyó la versión final aprobada que recogió los contenidos temáticos propuestos. Se respetaron los principios éticos para este tipo de estudio.

\section{Resultados}

El programa de la especialidad de oftalmología tiene una estructura donde los elementos del macrocurrículo y microcurrículo constituyen la forma en que se organiza. Dentro del macrocurrículo se encuentran los datos generales, la fundamentación, el perfil del especialista, la caracterización del egresado y el perfil profesional. El microcurrículo incluye la estructura del plan de estudios, los objetivos generales, procederes y cirugías por años. El plan de estudio se organiza por años y funciones, donde 22 módulos dan salida a los objetivos de los tres años y los residentes obtendrán 158 créditos académicos. La estrategia docente y la bibliografía también integran esta área del currículo.

\subsection{Análisis de contenido del programa}

En la tabla 1 se presenta el análisis de las habilidades y contenidos que los especialistas en oftalmología cubano deben adquirir durante su formación. Para cada una de las funciones declaradas en el perfil de egresado - asistenciales e investigativa- se hace referencia de forma genérica a las habilidades de promoción, prevención, diagnóstico, tratamiento clínico, médico de las complicaciones, así como de investigación para el desarrollo final del trabajo de terminación de residencia. Fue interesante que dentro de las habilidades y contenidos declarados no incluyeron los relacionados con el área de conocimiento de la farmacoepidemiología (tabla 1). 
Tabla 1. Funciones del especialista de oftalmología según lo declarado en el programa.

\begin{tabular}{|c|c|}
\hline & Habilidad y contenido \\
\hline Asistencial & $\begin{array}{l}\text {-Adquirir conocimientos y habilidades relacionados con su formación básica de los } \\
\text { problemas generales comunes a todas las enfermedades clínico/quirúrgicas } \\
\text { oftalmológicas... } \\
\text {-Aprender a diagnosticar y tratar las enfermedades ... } \\
\text {-Valorar el resultado del tratamiento, el seguimiento de los pacientes... } \\
\text {-Diagnosticar y tratar las enfermedades más frecuentes de la úvea, del cuerpo vítreo y la } \\
\text { retina... } \\
\text {-Seguir el postoperatorio de los pacientes intervenidos quirúrgicamente ... } \\
\text {-Abordar las manifestaciones oculares de las enfermedades sistémicas más comunes } \\
\text {-Aprender el manejo de la clínica neurológica para el diagnóstico, pronóstico, tratamiento } \\
\text { clínico y el seguimiento de los casos quirúrgicos. } \\
\text {-Diagnosticar los defectos refractivos más frecuentes, tratamiento quirúrgico y valorar } \\
\text { adecuadamente el resultado del tratamiento y manejo de las posibles complicaciones. } \\
\text {-Realizar acciones de promoción y prevención sobre el niño, la familia y la comunidad... } \\
\text {-Diagnosticar las enfermedades oculares más frecuentes en la edad pediátrica y aplicar } \\
\text { tratamiento integral }\end{array}$ \\
\hline Investigativo & $\begin{array}{l}\text { - Investigar en su práctica como especialista en Oftalmología y elaborar proyecto de } \\
\text { investigación } \\
\text {-Defender en el Examen Estatal los resultados de la investigación }\end{array}$ \\
\hline
\end{tabular}

En el análisis por años de la especialidad y su organización por módulos, objetivos y contenidos se hace referencia solo a los aspectos relacionados con la farmacología de los medicamentos en el tratamiento médico y quirúrgico durante los diferentes momentos del proceso de atención. En las tablas 2, 3 y 4 se reflejan los resultados del análisis de contenido por años de la especialidad y su organización por módulos, así como los objetivos y contenidos en cada uno de estos. En ellos se hace referencia a los aspectos que pudieran estar relacionados con la farmacoepidemiología. De forma general se observa que los elementos de coincidencia se refieren a tratamiento médico quirúrgico en los diferentes momentos del proceso de atención.

Un aspecto interesante en los siete módulos del primer año de la especialidad (pre operatorio y post operatorio, urgencias no traumáticas más frecuentes, urgencias traumáticas más frecuentes, cirugía estética y oculoplastia (COP), córnea, glaucoma y catarata) fue que en los objetivos confluyeron las unidades de sentidos de los aspectos relacionados con el tratamiento médico específicos de cada una de las enfermedades, asicomo la valoración del resultado del tratamiento y sus complicaciones; en este sentido, se hace referencia a la aplicación de diferentes técnicas de anestesia en oftalmología, asi como a la aplicación del tratamiento adecuado en las diferentes afecciones no traumáticas oculares; este último objetivo estuvo presente en las diferentes afecciones traumáticas oculares, las enfermedades de las vías lagrimales y párpados, el glaucoma de acuerdo con el diagnóstico y el pronóstico y las enfermedades del cristalino (tabla 2).

Un aspecto que estuvo presente en los módulos de córnea y enfermedades del cristalino fue la valoración del resultado de los tratamientos y las complicaciones; en este sentido se hace referencia al objetivo de "Valorar las complicaciones de acuerdo con el diagnóstico, la prevención y el tratamiento en los pacientes con glaucoma", "el resultado del tratamiento y el seguimiento de los pacientes con enfermedades del cristalino".

Los contenidos reflejan la proyección de los objetivos que fueron diseñados para este año de la especialidad. Estos respondieron a los aspectos clínicos-farmacológicos del 
tratamiento médico y carecen del enfoque sobre prescripción basada en evidencias y razonada, farmacovigilancia, reacciones adversas, pauta terapéutica y adherencia terapéutica. Los principales contenidos estuvieron dirigidos a "Tipos de anestesia que se aplican en la cirugía ocular; anestesia local periorbitaria, retrobulbar, intracraneal", "Acción terapéutica de urgencia: profilaxis de la infección" y "Conducta terapéutica en traumatismo abiertos"; este contenido estuvo presente en las queratoconjuntivitis, las enfermedades del aparato lagrimal, de la córnea, las Cataratas y el Glaucoma, en este último se hace referencia a las drogas locales y sistémicas y los diferentes grupos de medicamentos. El único lugar donde se hace referencia a las reacciones adversas fue en el módulo de córnea y glaucoma y en estos los contenidos incluyen: "Reacciones tóxicas y alérgicas a medicamentos oftálmicos tópicos" y en glaucoma tienen incluido "Indicaciones. Contraindicaciones. Efectos secundarios de los medicamentos".

Tabla 2. Contenidos del programa de formación del oftalmólogo cubano de $1^{\circ}$ año.

\begin{tabular}{|c|c|c|}
\hline Módulos & Objetivos & Contenidos \\
\hline $\begin{array}{l}\text { Preoperatorio y } \\
\text { Postoperatorio }\end{array}$ & $\begin{array}{l}\text { Aplicar diferentes técnicas de anestesia en } \\
\text { oftalmología. Prevenir, diagnosticar y curar } \\
\text { las complicaciones aplicando los criterios de } \\
\text { alta, determinando el grado. }\end{array}$ & $\begin{array}{l}\text { Medidas preopreparatorias del } \\
\text { paciente oftalmológico quirúrgico. } \\
\text { Tipos de anestesia en cirugía ocular. } \\
\text { Anestesia Local periorbitaria, } \\
\text { retrobulbar, intracraneal. Indicadores a } \\
\text { en la evaluación clínica }\end{array}$ \\
\hline $\begin{array}{l}\text { Urgencias no } \\
\text { Traumáticas más } \\
\text { frecuentes }\end{array}$ & $\begin{array}{l}\text { Aplicar tratamiento adecuado en las } \\
\text { diferentes afecciones no traumáticas } \\
\text { oculares. }\end{array}$ & $\begin{array}{l}\text { Acción terapéutica de urgencia: } \\
\text { profilaxis de la infección. }\end{array}$ \\
\hline $\begin{array}{l}\text { Urgencias } \\
\text { Traumáticas más } \\
\text { frecuentes }\end{array}$ & $\begin{array}{l}\text { Realizar tratamiento adecuado en las } \\
\text { diferentes afecciones traumáticas oculares. }\end{array}$ & $\begin{array}{l}\text { Conducta terapéutica ante cada tipo de } \\
\text { traumatismo abierto. Conducta a } \\
\text { seguir. Queratoconjuntivitis actínica } \\
\text { tratamiento }\end{array}$ \\
\hline $\begin{array}{l}\text { Cirugía estética y } \\
\text { oculoplastia }\end{array}$ & $\begin{array}{l}\text { Aplicar tratamiento integral a pacientes con } \\
\text { enfermedades de las vías lagrimales y } \\
\text { párpados, teniendo en cuenta el tratamiento }\end{array}$ & $\begin{array}{l}\text { Enfermedades del aparato lagrimal. } \\
\text { Tratamiento. } \\
\text { conjuntival: manejo y preparación pre } \\
\text { y post operatoria. }\end{array}$ \\
\hline Córnea & $\begin{array}{l}\text { Aplicar atención integral a pacientes con } \\
\text { enfermedades de la córnea y superficie } \\
\text { externa ocular, teniendo en cuenta el } \\
\text { tratamiento. Valorar el resultado del } \\
\text { tratamiento y el seguimiento de los pacientes } \\
\text { con enfermedades de la córnea }\end{array}$ & $\begin{array}{l}\text { Fármacos de uso frecuente en las } \\
\text { enfermedades de la córnea. Reacciones } \\
\text { toxicas y alérgicas a medicamentos } \\
\text { oftálmicos tópicos. }\end{array}$ \\
\hline Glaucoma & $\begin{array}{l}\text { Aplicar tratamiento en pacientes con } \\
\text { glaucoma de acuerdo con el diagnóstico y el } \\
\text { pronóstico. Valorar las complicaciones de } \\
\text { acuerdo con el diagnóstico, la prevención y } \\
\text { el tratamiento en los pacientes con glaucoma. }\end{array}$ & $\begin{array}{l}\text { Glaucoma agudo. Tratamiento. } \\
\text { Farmacología en el glaucoma: Drogas } \\
\text { locales y sistémicas. Grupos de } \\
\text { medicamentos. Medicamentos. } \\
\text { Mecanismo de acción. Indicaciones. } \\
\text { Contraindicaciones. } \\
\text { secundarios. }\end{array}$ \\
\hline Catarata & $\begin{array}{l}\text { Aplicar atención integral a pacientes con } \\
\text { enfermedades del cristalino, teniendo en } \\
\text { cuenta el tratamiento. Valorar el resultado } \\
\text { del tratamiento y el seguimiento de los } \\
\text { pacientes con enfermedades del cristalino }\end{array}$ & $\begin{array}{l}\text { Anomalías de posición: ectopias y } \\
\text { luxaciones. Conducta. Catarata } \\
\text { complicada y glaucomas de origen } \\
\text { cristaliniano. Cataratas secundarias a } \\
\text { enfermedades oculares y sistémicas. } \\
\text { Conducta a seguir. }\end{array}$ \\
\hline
\end{tabular}


En el segundo año de la especialidad (tabla 3) en el diseño curricular se encuentran seis módulos, el de Úvea y Retina, Manifestaciones Oculares de Enfermedades Sistémicas y se introducen nuevos objetivos y contenidos en los módulos de Oculoplastia, Córnea, Catarata y Glaucoma. Un elemento que fue común a todos los módulos fue el abordaje de contenidos en el tratamiento clínico y quirúrgico.

Tabla 3. Contenidos del programa de formación del oftalmólogo cubano de $2^{\circ}$ año.

\begin{tabular}{|c|c|c|}
\hline Módulos & Objetivos & Contenidos \\
\hline Úvea, Retina & $\begin{array}{l}\text { Aplicar atención integral a } \\
\text { pacientes con enfermedades de la } \\
\text { úvea, teniendo en cuenta el } \\
\text { tratamiento. Valorar el resultado } \\
\text { del tratamiento y el seguimiento de } \\
\text { los pacientes con enfermedades del } \\
\text { cristalino. Aplicar atención integral } \\
\text { a pacientes con enfermedades del } \\
\text { cuerpo vítreo y de la retina, } \\
\text { teniendo en cuenta el tratamiento. } \\
\text { Valorar el resultado del tratamiento } \\
\text { y el seguimiento de los pacientes } \\
\text { con enfermedades del cuerpo vítreo } \\
\text { y de la retina ... }\end{array}$ & $\begin{array}{l}\text { Uveítis inespecífica. Tratamiento. Uveítis } \\
\text { específicas. Manifestaciones clínicas y } \\
\text { tratamiento. Desprendimiento de retina: } \\
\text { Regmatógeno y no regmatógeno. Tratamiento. }\end{array}$ \\
\hline $\begin{array}{l}\text { Manifestaciones } \\
\text { Oculares de } \\
\text { Enfermedades } \\
\text { Sistémicas }\end{array}$ & $\begin{array}{l}\text { Aplicar tratamiento integral a } \\
\text { pacientes con manifestaciones } \\
\text { oculares de las enfermedades } \\
\text { sistémicas teniendo en cuenta el } \\
\text { tratamiento. Valorar el resultado } \\
\text { del tratamiento y la conducta } \\
\text { posterior de acuerdo con el } \\
\text { tratamiento y la prevención en las } \\
\text { complicaciones. }\end{array}$ & $\begin{array}{l}\text { Efectos secundarios de la terapia } \\
\text { antineoplásica. Toxicidad ocular por fármacos } \\
\text { y drogas. }\end{array}$ \\
\hline Oculoplastia. & $\begin{array}{l}\text { Aplicar diagnóstico y tratamiento } \\
\text { integral a pacientes con } \\
\text { enfermedades de las vías } \\
\text { lagrimales y orbita. }\end{array}$ & $\begin{array}{l}\text { Celulitis orbitaria. Tratamiento. } \\
\text { Fracturas orbitarias. Tratamiento. } \\
\text { Tumores de la Órbita. Tratamiento. }\end{array}$ \\
\hline Córnea. & $\begin{array}{l}\text { Realizar el diagnóstico y } \\
\text { tratamiento de las enfermedades de } \\
\text { la córnea y superficie externa } \\
\text { ocular. }\end{array}$ & $\begin{array}{l}\text { Queratitis. Queratitis virales, bacterianas, } \\
\text { fúngicas y parasitarias. Cuadro clínico. } \\
\text { Conducta y seguimiento. Degeneraciones } \\
\text { corneales. Arco senil, degeneración lipídica, } \\
\text { amiloide, esferoidal, nodular de Salzman, } \\
\text { keloide corneal, marginal de Terrien, } \\
\text { Queratopatía en banda. Conducta. } \\
\text { Pigmentaciones corneales. Huso de } \\
\text { Krukenberg, coloración hemática, anillo de } \\
\text { Kayser- Fleischer, líneas de hierro, por } \\
\text { depósitos de medicamentos.Conducta. } \\
\text { Distrofias corneales y ectasias. Distrofias } \\
\text { epiteliales, estromales y endoteliales. } \\
\text { Queratocono. Conducta. Enfermedades corneo } \\
\text { conjuntivales mediadas por trastornos } \\
\text { inmunológicos: Síndrome de Stevens-Johnson, } \\
\text { Penfigoide cicatrizal ocular, queratitis }\end{array}$ \\
\hline
\end{tabular}




\begin{tabular}{|c|c|c|}
\hline & & $\begin{array}{l}\text { punteada superficial de Thygenson, queratitis } \\
\text { ulcerativas periféricas, ulcera de Mooren. } \\
\text { Conducta. Insuficiencia de células madres del } \\
\text { limbo. Conducta. Traumatología corneal. } \\
\text { Heridas corneales, cuerpos extraños corneales, } \\
\text { quemaduras corneo conjuntivales Tratamiento } \\
\text { médico- quirúrgico. Introducción general al } \\
\text { Trasplante Corneal. Conducta a seguir. }\end{array}$ \\
\hline Catarata & $\begin{array}{l}\text { Seguimiento postoperatorio del } \\
\text { paciente operado de catarata. } \\
\text { Conocer el seguimiento clínico y el } \\
\text { tratamiento quirúrgico de las } \\
\text { principales complicaciones. }\end{array}$ & $\begin{array}{l}\text { Uveítis postquirúrgica. Tratamiento. } \\
\text { Endoftalmitis. Tratamiento. }\end{array}$ \\
\hline Glaucoma & $\begin{array}{l}\text { Diagnosticar precozmente el } \\
\text { glaucoma congénito aplicando } \\
\text { tratamiento. Aplicar tratamiento } \\
\text { médico- quirúrgico al glaucoma } \\
\text { congénito teniendo en cuenta el } \\
\text { diagnóstico, el pronóstico y los } \\
\text { procederes que correspondan en su } \\
\text { nivel de atención. }\end{array}$ & $\begin{array}{l}\text { Glaucoma Primario de ángulo abierto: } \\
\text { Tratamiento. Glaucoma Secundario: y } \\
\text { refractarios. Conducta a seguir. Glaucoma } \\
\text { Primario o infantil: Conducta a seguir. } \\
\text { Glaucoma Asociado a anomalías congénitas: } \\
\text { Tratamiento. Glaucoma Secundario: } \\
\text { Tratamiento. }\end{array}$ \\
\hline
\end{tabular}

Los principales objetivos tuvieron un enfoque similar a los que se desarrollaron en el primer año de la residencia donde estuvieron dirigido a aplicar tratamiento en las diferentes enfermedades y en algunos casos valorar el resultado del tratamiento, el seguimiento postquirúrgico de los casos y sus complicaciones. Las principales unidades de sentido identificadas en el análisis fueron: Aplicar atención integral a pacientes con enfermedades de la úvea, teniendo en cuenta el tratamiento, este mismo objetivo se planteó para los módulos que abordaron las enfermedades del cuerpo vítreo y de la retina, las enfermedades sistémicas, de enfermedades de las vías lagrimales y órbita y glaucoma congénito. Los contenidos abordaron el tratamiento médico y quirúrgico como se planteó en los objetivos. Sin embargo, no se realiza un abordaje de aspectos importantes de la farmacoepidemiología en el proceso formación, lo cual se refleja a través de los diferentes contenidos formulados donde se hace referencia al tratamiento de las diferentes enfermedades como las uveítis inespecífica y especifica. La perspectiva de formulación de los objetivos está presente al abordar los contenidos del resto de las enfermedades como el desprendimiento de retina regmatógeno y no regmatógeno, celulitis orbitaria, fracturas orbitaria, tumores de la órbita, queratitis, pigmentaciones corneales, distrofias corneales y ectasias, epiteliales, estromales y endoteliales, queratocono, enfermedades corneo conjuntivales, traumatología corneal y trasplante corneal, las uveítis postquirúrgica, las endoftalmitis y los diferentes tipos de glaucoma. En el módulo de manifestaciones oculares de enfermedades sistémicas fue donde único existieron contenidos que pudiera estar relacionada con la prescripción basa en evidencia en pacientes con neoplasias, esto se constató al tratar los contenidos de los efectos secundarios de la terapia antineoplásica y Toxicidad ocular por fármacos y drogas.

El programa de la especialidad en el tercer año (tabla 4) incluye seis módulos, el de vitro-retina, neuro-oftalmología, cirugía refractiva, catarata, prevención oftalmológica en el niño y enfermedades oftalmológicas en el niño. Continúan el diseño de los objetivo con la misma perspectiva del primer y segundo año del programa de la especialidad, estos responden a los niveles de aprendizaje de conocimiento, aplicación y estan enfocados al tratamiento clínico-quirúrgico específico y el seguimiento de los pacientes y sus complicaciones. 
Tabla 4. Contenidos del programa de formación del oftalmólogo cubano de $3^{\circ}$ año.

\begin{tabular}{|c|c|c|}
\hline Módulos & Objetivos & Contenidos \\
\hline Vitro-Retina & $\begin{array}{l}\text { Aplicar atención integral a } \\
\text { pacientes con enfermedades del } \\
\text { cuerpo vítreo y de la retina, } \\
\text { teniendo en cuenta el tratamiento. } \\
\text { Valorar el resultado del } \\
\text { tratamiento y el seguimiento de } \\
\text { los pacientes con enfermedades } \\
\text { del cuerpo vítreo y de la retina }\end{array}$ & $\begin{array}{l}\text { Patología general del vítreo: } \\
\text { Desprendimiento posterior del vítreo. } \\
\text { Tratamiento. Hemorragia vítrea.. } \\
\text { Endoftalmitis. Tratamiento. Desprendimiento de } \\
\text { retina: Regmatógeno y no regmatógeno. } \\
\text { Tratamientos. Enfermedades Vasculares de la } \\
\text { Retina.. Tratamiento. Tumores de retina. } \\
\text { Tratamiento. }\end{array}$ \\
\hline $\begin{array}{l}\text { Neuro- } \\
\text { Oftalmología }\end{array}$ & $\begin{array}{l}\text { Aplicar el manejo de la clínica } \\
\text { neurológica para el tratamiento } \\
\text { clínico y el seguimiento de los } \\
\text { casos quirúrgicos }\end{array}$ & No refiere \\
\hline $\begin{array}{l}\text { Cirugía } \\
\text { Refractiva }\end{array}$ & $\begin{array}{lll}\text { Valorar adecuadamente el } \\
\text { resultado del tratamiento y } \\
\text { manejo de las posibles } \\
\text { complicaciones. }\end{array}$ & $\begin{array}{l}\text { Manejo post operatorio. Postoperatorio normal: } \\
\text { tratamiento. }\end{array}$ \\
\hline Catarata & $\begin{array}{l}\text { Aplicar tratamientos quirúrgicos a } \\
\text { pacientes con enfermedades del } \\
\text { cristalino, e indicar rehabilitación } \\
\text { óptica. } \\
\text { Conocer el seguimiento clínico y } \\
\text { el tratamiento quirúrgico de las } \\
\text { principales complicaciones }\end{array}$ & $\begin{array}{l}\text { Manejo de las principales complicaciones } \\
\text { derivadas de la cirugía de catarata. Uveítis } \\
\text { postquirúrgicaTratamiento. } \\
\text { Tratamiento. }\end{array}$ \\
\hline $\begin{array}{l}\text { Prevención } \\
\text { Oftalmológica en } \\
\text { el niño }\end{array}$ & $\begin{array}{l}\text { Aplicar tratamiento a pacientes } \\
\text { con ambliopía teniendo en cuenta } \\
\text { la clasificación, el pronóstico y el } \\
\text { nivel de atención del servicio en } \\
\text { que se desempeñe. }\end{array}$ & $\begin{array}{l}\text { Ambliopía. Tratamiento de acuerdo al nivel de } \\
\text { atención. }\end{array}$ \\
\hline $\begin{array}{l}\text { Enfermedades } \\
\text { Oftalmológicas en } \\
\text { el niño }\end{array}$ & $\begin{array}{l}\text { Aplicar tratamiento integral al } \\
\text { paciente pediátrico con } \\
\text { enfermedades oftalmológicas, } \\
\text { teniendo en cuenta el tratamiento. } \\
\text { Valorar el resultado del } \\
\text { tratamiento y la conducta } \\
\text { posterior. }\end{array}$ & $\begin{array}{l}\text { Obstrucción congénita del conducto nasolagrimal. } \\
\text { Tratamiento. Glaucoma congénito.Tratamiento. } \\
\text { Esotropías. Tratamiento. Exotropías. Tratamiento. } \\
\text { D.V.D y D.H.D. Tratamiento. Anisotropías. } \\
\text { Tratamiento. Paresias y Parálisis oculomotoras: } \\
\text { Tratamiento. Estrabismos restrictivos: Tratamiento. } \\
\text { Síndromes especiales de la motilidad ocular: } \\
\text { Tratamiento. Nistagmo. Tratamiento Retinopatía } \\
\text { de la prematuridad activa. } \\
\text { Tratamiento. Retinopatía de la prematuridad } \\
\text { cicatrizal. Tratamiento. }\end{array}$ \\
\hline
\end{tabular}

En este año, los objetivos se dirigen a la aplicación de la atención integral a pacientes con enfermedades de la úvea, teniendo en cuenta el tratamiento; de esta misma forma se encuentran formulados en los módulos de las enfermedades de cuerpo vítreo y de la retina, las enfermedades sistémicas, de vías lagrimales y orbita. Existen otros objetivos dirigidos a valorar el resultado del tratamiento y seguimiento de los pacientes con enfermedades del cuerpo vítreo y de la retina y en paciente pediátrico con enfermedades oftalmológicas, también están los que plantean el conocimiento del seguimiento clínico y tratamiento quirúrgico de las principales complicaciones de enfermedades del cristalino, así como la indicación de la rehabilitación óptica. En este año de la residencia se constata la correspondencia entre los objetivos y contenidos. Estos se plantearon hacia el tratamiento 
médico-quirúrgico y seguimiento de enfermedades general del vítreo, las endoftalmitis, el desprendimiento de retina regmatógeno y no regmatógeno, las enfermedades vasculares de la retina, los tumores de retina, las uveítis postquirúrgica, la ambliopía, obstrucción congénita del conducto nasolagrimal, glaucoma congénito, esotropías. exotropía, anisotropías. paresias y parálisis oculomotoras, estrabismos restrictivos, síndromes especiales de la motilidad ocular, nistagmo, retinopatía de la prematuridad activa, retinopatía de la prematuridad cicatrizal.

\subsection{Definición de contenidos por grupo de expertos}

En la técnica participaron 7 expertos con las características descrita en la metodología, lográndose el 100 \% de participación durante la técnica. En la etapa de consulta se logró el objetivo propuesto de consenso del $85 \%$ de los expertos con relación a los contenidos. En la primera ronda se plantearon 12 temas a incluir en los diferentes momentos del programa, en la segunda ronda se redujeron a 10 temas y en la tercera ronda quedaron 9 temas. Los temas que no se incluyeron fueron el de farmacoeconomía, estrategia de marketing de medicamentos, metanálisis de medicamentos. A través del consenso derivado de la consulta a los expertos se definieron los contenidos temáticos a incluir en el programa. Los mismos consideraron de manera general los elementos de prescripción y farmacovigilancia como imprescindibles en la formación del oftalmólogo cubano. A continuación se presentan los mismos (tabla 5).

Tabla 5. Temas de farmacoepidemiología seleccionados por los expertos ( $n=7)$.

\begin{tabular}{|l|c|c|c|}
\hline \multicolumn{1}{|c|}{ Temas de Farmacoepidemiología } & \% & Media & $\begin{array}{c}\text { Desviación } \\
\text { estándar }\end{array}$ \\
\hline Lectura critica & 100 & 9,1 & 0,9 \\
\hline Selección de fármaco & 85,7 & 7,4 & 1,9 \\
\hline Prescripción basada en evidencias & 100 & 8,7 & 0,8 \\
\hline Farmacovigilancia & 100 & 8,7 & 0,8 \\
\hline Reacciones adversas & 100 & 8,8 & 0,7 \\
\hline Prescripción razonada & 85.7 & 7,7 & 1,8 \\
\hline Pauta terapéutica & 85.7 & 7,8 & 1,7 \\
\hline Adherencia terapéutica & 100 & 9,0 & 0,8 \\
\hline Comité farmacoterapéutico & 100 & 9,1 & 0,9 \\
\hline
\end{tabular}

Se pudo observar que los expertos dieron mayor puntuación a siete temas; los mayores pocentajes fueron para la lectura crítica, la prescripción basada en evidencias, la farmacovigilancia, las reacciones adversas, la adherencia terapéutica y el comité farmacoterapéutico; para estos, la media de puntos fue entre 8,7 y 9,1 y la desviación estándar entre 0,7 y 0,9, clasificándose dentro de la categoría muy importante. Los contenidos relacionados con la selección de fármaco, la prescripción razonada y la pauta terapéutica tuvieron un porcentaje del $85,7 \%$, con una media de 7,4 a 7,7 con una desviación estándar entre 1,7 y 1,9 y fueron considerados como importantes.

\section{Discusión}

La investigación educacional, la crítica científica, el análisis minucioso de las experiencias de implementación de los modelos de formación son esenciales para seguir 
adelante en la obtención de un escenario de formación cada vez más sólido. En el análisis de contenido del programa de formación del especialista en oftalmología cubano, se pudo apreciar que están declaradas las habilidades y contenidos por cada una de las funciones declaradas en el perfil del egresado (asistenciales, investigativas, docentes y de dirección) en la función asistencial fue donde mayor número de habilidades se declararon. Estas transitan desde los aspectos generales de las enfermedades clínicas y quirúrgicas hasta específicos para prevenir, diagnosticar, tratar, curar y rehabilitar las afecciones de los segmentos anterior y posterior, del nervio óptico y de anexos oculares. Llama la atención que no se hace referencia de forma explícita a elementos que puedan indicar la formación de habilidades en farmacoepidemiología. Se pudo apreciar, como regularidad, que se hace mención en los objetivos y contenidos de los módulos que tributan a la función asistencial los términos tratamiento y conducta como elemento que vincula la formación en el área de farmacoepidemiología. Esto deja a la espontaneidad del profesor la introducción de aspectos básicos relacionados con la prescripción, consumo de medicamentos, reacciones adversas a medicamentos, pauta terapéutica y la farmacovigilancia, entre otros, necesarios durante la formación de este especialista para lograr las competencias y la calidad de la atención oftalmológica.

Como consecuencia, las prácticas prescriptivas son irracionales e incoherentes, basadas en la experiencia personal de los docentes (que por demás puede ser muy corta y limitada) y donde se pueden encontrar errores como tratamientos innecesarios, duración errónea, profilaxis mal orientada y selección incorrecta del medicamento, que son apropiadas por el estudiante como prácticas adecuadas y usadas al término de sus estudios sin un criterio propio (6). Los autores consideran como positivo que desde el primer año de la especialidad en el módulo de glaucoma se hace referencia a la farmacología de los medicamentos, el uso de drogas locales y sistémicas, los grupos de medicamento, su mecanismo de acción, indicaciones, contraindicaciones, efectos secundarios. En el segundo año en el módulo de manifestaciones oculares de enfermedades sistémicas se tienen en cuenta los efectos secundarios de la terapia antineoplásica, así como la toxicidad ocular por fármacos y drogas. De esta forma se evidencia que existe en la formación del oftalmólogo cubano una aproximación en estos módulos a los conocimientos y habilidades que se necesita de farmacoepidemiología.

En los módulos de baja visión y neuroftalmología no se hacen referencia a objetivos y contenidos relacionados con el área de conocimiento. En neuroftalmología existen enfermedades que son causadas por el uso de medicamentos, y otras donde estos deben ser controlados debido a sus efectos adversos (7-9). Llama la atención que en el programa no se tienen en cuenta la formación habilidades para prevenir y tratar estas enfermedades oftalmológicas. Los módulos que tributan a las funciones docente, de investigación y dirección no hace referencia en sus objetivos y contenido a esta área del conocimiento, pero a pesar de esto, se pueden desarrollar proyectos de investigación y un sistema de vigilancia de reacciones adversas a medicamentos, dispositivos, soluciones, entre otros aspectos, que puedan contribuir al proceso de formación. Además resulta útil en el área gerencial, la incorporación del funcionamiento del comité farmacoterapéutico como órgano asesor para lograr el uso adecuado de los medicamentos. A juicio de los autores, para atenuar las dificultades observadas se hace necesario un perfeccionamiento de los programas de formación del oftalmólogo sobre farmacoepidemiología, un uso eficiente de las actividades de educación continuada como cursos, así como estrategias de gestión para garantizar una farmacovigilancia efectiva en los servicios, dentro de éstas últimas están el seguimiento y la supervisión de la actividad por un responsable en cada servicio, con apoyo institucional. 
Existe en la actualidad la necesidad de crear una cultura del reporte de reacciones adversas a medicamentos, de generar un conocimiento relativo a ésta disciplina y de integrar a los oftalmólogos al sistema nacional de farmacovigilancia, el cual articule a un tiempo difusión y educación, y realización de investigaciones basadas en el método epidemiológico. A decir de García Milian (10), se necesitan conocer las ventajas e inconvenientes del uso de los medicamentos mediante estudios que muestren en qué pacientes se utilizan y en qué condiciones, cómo se utilizan, por qué se utilizan, qué efectos tienen, qué factores condicionan esos efectos y qué costo tienen en las condiciones habituales de la práctica clínica, de tal forma que permita a los gerentes desarrollar intervenciones para intentar resolver estos problemas. Carnota Lauzan (11) plantea que la propia dinámica de los paradigmas y conocimientos en materia de salud y los nuevos enfoques en salud pública, obligan a reestructurar, cada vez en períodos más cortos, la formación pre y posgraduada de los profesionales sanitarios. Continúa diciendo que para lograr el uso adecuado de los medicamentos en términos de prescripción, seguridad y consumo se torna vital la implementación de una política de calidad, tanto en la formación, como del sistema de salud en general. De manera que se involucre al personal técnico, las autoridades y las universidades.

La farmacoepidemiología enseña a obtener información confiable e independiente, lo que le permite al médico una lectura crítica de artículos científicos y llegar a sus propias conclusiones (12). En este sentido los expertos se pronunciaron con la incorporación de estas habilidades en la especialidad de oftalmología. La formación de un pensamiento crítico y de habilidades de lectura crítica tiene importancia reconocida como competencias a desarrollar en el ámbito educacional (13). La lectura de textos puede estar motivada y orientada por diferentes objetivos y metas: obtener, comprender, interpretar o construir el propio conocimiento; aún más, analizar la información, sintetizarla, interpretarla, criticarla y trascenderla en su uso y aplicación en la solución de problemas, en este caso, problemas de salud de la población (14). En el proceso de formación, la lectura crítica es estrategia, método y técnica, según su nivel de abordaje, lo que le permite al alumno de medicina y al profesor tener información adecuada para construir su propio conocimiento y desarrollar competencias técnico-médicas y sociohumanistas, a fin de mejorar el desempeño en la atención de los pacientes. La lectura crítica es indispensable para el aprendizaje independiente y la toma de decisiones objetivas, válidas y científicamente documentadas (15). Botello Sánchez y Alonso Marín (16) realizaron un estudio sobre la motivación de los estudiantes cubanos de Humanidades por la lectura crítica durante la carrera y plantean que esta tributa a una mejoría del interés y de las habilidades relativas a la emisión de juicios, valoraciones y modos de actuación.

En cuanto a la selección de medicamentos, su práctica adecuada tributa no solo a elevar la calidad de la atención médica sino a su gestión y la eficiencia de los recursos sanitarios. A esto se le suma que el proceso de selección de medicamentos puede resultar en una guía de práctica clínica o recomendaciones que ayuden al médico en la elaboración de su formulario personal (17). La selección del medicamento dependerá en buena medida, del proceso que ha llevado a decidir su utilización, y el resultado final de su aplicación dependerá además, de cómo el paciente lo utilice. Las decisiones que se toman por parte de los profesionales y los gerentes sanitarios deben ser cada vez más racionales y basadas en las evidencias científicas. Por tanto se hace necesario transformar los modelos de decisiones tradicionales basados en criterios subjetivos (autoridad profesional, experiencia clínica) como fuente de información, hacia modelos actuales fundamentados en pruebas científicas (evidencias). La falta de actualización constante lleva al médico a un progresivo deterioro de su competencia después de terminar su formación (18). 
Los fallos al seguir las prescripciones médicas exacerban los problemas de salud y la progresión de las enfermedades, haciendo imposible estimar los efectos y el valor de un determinado tratamiento. Esto imposibilita que se realice un buen diagnóstico y provoca un aumento innecesario del costo de la atención sanitaria tanto para el paciente como para el sistema de salud. Teniendo en cuanta los elementos antes mencionados se justifica la inclusión del tema de prescripción razonada en general y de pauta terapéutica en particular sugerida por los expertos. La adherencia terapéutica es otro de los contenidos que estos recomiendan incorporar a la especialidad. La OMS asegura que el incumplimiento del tratamiento es la principal causa de no obtención de los beneficios que los medicamentos pueden proporcionar. En los países desarrollados la adherencia terapéutica en pacientes con enfermedades crónicas es solo 50 por ciento y se supone que esta deficiencia sea mayor en países en desarrollo, dada la escasez de recursos y las iniquidades en el acceso a la atención (19). Se considera como la respuesta positiva del paciente a la prescripción realizada por el facultativo, dado el sentido psicológico que este entraña. Este fenómeno está condicionado por múltiples factores de diversa naturaleza. Entre ellos se mencionan los relacionados con el paciente, con el profesional, con el sistema sanitario, con la enfermedad y con los propios fármacos. Por tal razón los autores consideran oportuno incorporar este tema en la maya curricular del oftalmólogo. Lo antes dicho se justifica por las repercusiones que el incumplimiento tiene desde el punto de vista clínico, médico, económico y psicosocial. El mismo afecta cuestiones que tienen que ver con la calidad de la atención, la relación médico-paciente, el uso racional de los recursos y los servicios de salud, entre otros.

El Comité Farmacoterapéutico es un foro que reúne a las personas implicadas en la prestación de servicios de salud y constituye un instrumento para fomentar el uso adecuado de los medicamentos. Su propósito principal es asesorar en la selección de medicamentos, monitorear su utilización, entrenar, recolectar información sistematizada para adoptar programas y estrategias nuevas (20-21). En tal sentido, una gestión óptima del Comité Farmacoterapéutico puede mejorar de forma significativa el uso de medicamentos y reducir los costos en los centros de atención de salud, lo que asegura una asistencia médica de calidad con el menor costo posible (22). A juicio de los autores, la realización de modalidades capacitantes que vinculen a la teoría con la formación de competencias, permite que los presciptores se interesen y apropien de la farmacoepidemiología como una herramienta para el uso racional de los medicamentos. El conocimiento de la farmacoepidemiología y sus posibilidades de aplicación, por los profesionales, amplia la visión de los mismos en el afrontamiento y solución de los problemas de la salud pública desde una perspectiva social. La formación en farmacoepidemiología posibilita minimizar los efectos perjudiciales de los medicamentos al proporcionar a las autoridades la información necesaria para corregir las recomendaciones sobre su uso. Esta convierte a los prescriptores en entes impulsores para la difusión de la información farmacoterapéutica independiente que se requiere, compulsa las acciones con el resto de los actores relacionados con los medicamentos, para así promover el uso adecuado de los medicamentos en la salud pública cubana.

\section{Conclusiones}

- En el programa de formación del oftalmólogo cubano existe poca sistematización de contenidos temáticos relacionados con la selección de medicamentos, prescripción razonada y su vigilancia, elementos estos que influyen en la formación de habilidades y competencias para su uso.

- La incorporación de los contenidos temáticos definidos por los expertos a la formación del especialista de oftalmología en Cuba guarda relación con la ausencia de 
estos en el programa y se sustentan en las necesidades de aprendizaje identificadas para elevar el desempeño profesional en farmacoepidemiología.

Financiación: No ha habido financiación.

Declaración de conflicto of interés: No existe conflicto de interés.

\section{Referencias}

1. Cires M. Estudios de utilización de medicamentos. En: Pérez Peña Furones JA. La Farmacoepidemiología como base científica para alcanzar un uso racional de los medicamentos en el sistema sanitario cubano. MINBAS_ MINSAP, Ciudad Habana, 2001; 5-13

2. Salas Perea RS, Díaz Hernández L, Pérez Hoz G. Las competencias y el desempeño laboral en el Sistema Nacional de Salud. Educ Med Super 2012; 26(4). http://scielo.sld.cu/scielo.php? script=sci_isoref\&pid=S0864-21412012000400013\&lng=es\&tlng=es

3. García Milian AJ, Galindo Reymod K, Morales Pérez M, León Cabrera P. Farmacovigilancia hospitalaria. Rev Cub Oftal 2016; 29(4): 61-70.

http://www.revoftalmologia.sld.cu/index.php/oftalmologia/article/view/443

4. Benitez Cartaya M, Cruz Barrio MA, Bayarre Vea H, Almenares Rodriguez K, Ambou Fruros I, Vilches Lescaille DC. Evaluación farmacoterapéutica y evolutiva de uveítis crónicas y recurrentes no infecciosas. Rev Cub Oftalm 2014; 27,(3). http://www.revoftalmologia.sld.cu/index.php/oftalmologia/article/view/ $\underline{322}$

5. García Milian AJ, Alonso Carbonell L, López Puig P, León Cabrera P, Segredo Pérez AM, Calvo Barbado DM. Propuesta metodológica para el análisis crítico a un programa de estudio. Educ méd sup. $2015 ; 29(2)$. http://www.ems.sld.cu/index.php/ems/article/view/382

6. Garcia Milian AJ. Investigación sobre el consumo de medicamentos, experiencia cubana. ECIMED. La Habana; 2013.p58-69

7. Pérez-Rodríguez E, Matheu-Delgado V, Sánchez-Machín I, García-Robaina JC, De la Torre-Morín F. Reacciones adversas durante la administración de fluoresceína endovenosa. Arch Soc Esp Oftalmol 2005; 80(8): 441-442. http:/ / scielo.isciii.es/scielo.php?script=sci_arttext\&pid=S0365-66912005000800003\&lng=es.

8. Rodríguez Vargas Gabriel. Efectos adversos oculares de fármacos sistémicos. Med. leg. Costa Rica 2013; 30(1): 117-122.

9. http://www.scielo.sa.cr/scielo.php?script=sci arttext\&pid=S1409-00152013000100014\&lng=en.

10. Gómez Escobar Luis Guillermo, González Devia Deyanira. Posibles efectos adversos oculares de los bifosfonatos. Reporte de 2 casos. Rev. Colomb. Reumatol 2017; 24(1): 54-59. http://dx.doi.org/10.1016/j.rcreu.2016.06.002.

11. García Milian AJ. Investigación sobre consumo de medicamentos. Experiencia cubana. La Habana; Ecimed; 2013. p 121.

12. Carnota Lauzán O. Hacia una conceptualización de la gerencia en salud a partir de las particularidades. Rev Cubana Salud Pública 2013; 39(3): 501-23. http://scielo.sld.cu/scielo.php? script=sci arttext\&pid=S0864-34662013000300008\&lng=es

13. Yero Alos I; Calvo D M, García Milian AJ. Manejo de la infección del tracto urinario. Rev Cub de Farm 2005; 39 (1). http://scielo.sld.cu/scielo.php?pid=S0034-5152005000100006\&script=sci abstract

14. Díaz JP, Bar AR, Ortiz MC. La lectura crítica y su relación con la formación disciplinar de estudiantes universitarios. Rev Educ Super. 2015; 44 (4):139-158.

15. http://www.scielo.org.mx/pdf/resu/v44n176/v44n176a7.pdf

16. García Vigil JL. Lectura crítica. Modelo de comunicación para el aprendizaje independiente y de la clínica. Rev Med IMSS 2000; 38 (1): 61-8. https://www.researchgate.net/profile/5527270c0cf2520617a70a5c/1

17. Broche Villarreal L, Furones Mourelle JA, Cruz Barrios MA, Rodríguez Piñeiro D. Composición y funcionamiento del comité farmacoterapéutico en policlínicos de La Habana. Rev Cub Sal Públ 2015;41(2): 239-52. http:// scielo.sld.cu/scielo.php?script=sci_arttext\&pid=S0864-34662015000200006\&lng=es

18. González Sánchez A. El desempeño profesional por competencias del tutor en la carrera de enfermería. [Tesis]. Universidad de Matanzas; 2016. 
19. Garjón Parra J. Evaluación y selección de medicamentos. Farmacéuticos de Atención Primaria 2011; 9 (3): 75-103. http://www.elsevier.es/es-revista-farmaceuticos-atencion-primaria-317-articulo-evaluacionseleccion-medicamentos-X2172376111012370

20. Garcia Milian AJ. La prescripción de fitofármacos basada en la evidencia. Rev Cub Plant Med 2019;23(3). http:/ / revplantasmedicinales.sld.cu/index.php/pla/article/view/877

21. Organización Mundial de la Salud. Adherencia a los tratamientos a largo plazo. Pruebas para la acción. Ginebra: OMS; 2004.

22. Green T, Beith A, Chalker J. Comités sobre medicamentos y terapéutica: vehículos para mejorar el uso racional de los medicamentos. Bol. Medicamentos Esenciales. 2003; 32: (10-1). http://apps.who.int/medicinedocs/es/d/Is5524s/6.html

23. Broche Villarreal L, Furones Mourelle JA, Cruz Barrios MA, Rodríguez Piñeiro D. Composición y funcionamiento del comité farmacoterapéutico en policlínicos de La Habana. Rev Cub Sal Públ. 2015; 41(2): 239-52. http://scielo.sld.cu/scielo.php?script=sci_arttext\&pid=S0864-34662015000200006\&lng=es

24. Furones Mourelle JA, Cruz Barrios MA, Broche Villarreal L, García Milian AJ, López Aguilera ÁF. Importancia de los comités farmacoterapéuticos en la toma de decisiones en salud. Rev INFODIR 2014 ; 0(17). http://revinfodir.sld.cu/index.php/infodir/article/view / 23

(c) 2021 por los autores. Enviado para su publicación en acceso abierto bajo los términos y condiciones de la licencia Creative Commons Attribution (CC BY) (http://creativecommons.org/licenses/by/4.0/). 